\title{
Effects of decreasing ionospheric pressure on the solar wind interaction with non-magnetized planets
}

\author{
T. Tanaka \\ Communications Research Laboratory, Koganei-shi, Tokyo 184, Japan \\ (Received August 1, 1997; Revised December 25, 1997; Accepted January 5, 1998)
}

\begin{abstract}
The large-scale solar wind interaction with the ionosphere of non-magnetized planets is numerically simulated in the framework of three-dimensional (3-D) magnetohydrodynamics (MHD) with a two-component plasma. The finite-volume total variation diminishing (TVD) scheme is used to solve this problem. Numerical results are given for two cases of different solar extreme ultraviolet (EUV) flux values. In case 1, solar EUV ionization is set so the peak ionospheric plasma pressure is below the incident solar wind dynamic pressure. In case 2, on the other hand, it is set so the peak ionospheric pressure exceeds the solar wind dynamic pressure. While the formation of the bow shock and the magnetic barrier in the upstream region is seen in both cases, a clear formation of the ionopause is seen only in case 2 . In case 1 , the interplanetary magnetic field (IMF) penetrates from the magnetosheath to the dayside ionosphere so as to adjust the ionospheric total pressure. Penetrating IMF affects the vertical motion of the ionospheric plasma to cause anomalous stratifications of the terminator ionosphere. However, formation process of the ionotail is little affected by the penetrating IMF. Another important process predicted from the present study is partial penetration of the IMF from the magnetic barrier to the terminator ionosphere. This nonideal MHD process characterized by the penetration of flowing magnetized plasma into non-magnetized plasma plays a principal role in the mixing interaction between the solar wind and the planetary ionosphere.
\end{abstract}

\section{Introduction}

In the process of the solar wind interactions with the nonmagnetized planets such as Mars and Venus, the highly conducting ionosphere directly contacts with the oncoming solar wind flow and deflects it around the planets (Luhmann and Brace, 1991). In such an interaction process, the supersonic solar wind flow recognizes the planetary ionosphere as an obstacle, and the bow shock forms upstream of the planets (Zhang et al., 1990; Slavin et al., 1991). The bow shock is a standing fast magnetosonic wave for an average solar wind condition.

In the solar wind, kinetic pressure dominates gas pressure and magnetic pressure. At the bow shock, the kinetic pressure of the solar wind is converted into thermal energy. The region between the bow shock and the ionosphere is, therefore, filled with high-temperature shocked plasma. This region is referred to as the magnetosheath (Phillips and McComas, 1991). In the inner region of the magnetosheath, the magnetic barrier, a region within which the magnetic pressure dominates all other pressure contributions, transfers thermal pressure in the magnetosheath to the ionosphere (Luhmann, 1986; Zhang et al., 1991). The formation of such magnetic barrier is a consequence of the draping process of the interplanetary magnetic field (IMF) around planets (Phillips et al., 1986; Tanaka, 1993). If the peak ionospheric plasma pressure exceeds the incident solar wind dynamic pressure, the ionopause, a sharp gradient in the plasma density, forms between the magnetic barrier and the iono-

Copy right (C) The Society of Geomagnetism and Earth, Planetary and Space Sciences (SGEPSS); The Seismological Society of Japan; The Volcanological Society of Japan; The Geodetic Society of Japan; The Japanese Society for Planetary Sciences. sphere and separates the magnetic barrier from the thermal ionospheric plasma (Phillips and McComas, 1991). This condition is analogous to the case of a superconducting sphere in a flowing magnetized plasma.

Under the condition of high-speed solar wind flow or low solar extreme ultraviolet (EUV) flux, the ionospheric plasma pressure can be below that of the upstream solar wind dynamic pressure. In this situation, the IMF penetrates the dayside ionosphere and supplements the total pressure with magnetic pressure (Luhmann et al., 1987; Luhmann and Cravens, 1991). Thus, the analogy with a superconducting sphere breaks down, and the status of imperfect shielding is realized. As a consequence, large scale horizontal magnetic fields will appear in both the dayside and nightside ionospheres. These magnetic fields can affect the formation process of both the dayside and nightside ionospheres (Shinagawa, 1996b). The nightside ionosphere extends tailward at least few thousand kilometers downstream and continues to the region called as the ionotail or tail ray (Brace et al., 1987). The formation process of the ionotail can be also affected by the magnetic field structure in the ionosphere. The purpose of this paper is to investigate how the imperfect shielding state modifies the solar wind-planetary ionosphere interaction from a theoretical investigation which adopts the 9-component magnetohydrodynamic (MHD) equation for a two-component reacting plasma (Tanaka and Murawski, 1997).

Although the MHD theory is the simplest self-consistent model describing the macroscopic behavior of the plasma, the complete nonlinear equations are so complex that drastic simplifications are usually necessary to make the problems 
tractable. Many solutions therefore require numerical treatment, but MHD equations tend to form shocks and discontinuities, which are difficult to treat numerically. The use of standard numerical schemes of second-order accuracy or higher (e.g., the Lax-Wendroff method) generates spurious oscillations which generally occur at high-gradients and consequently destroy the monotonicity of the solution. Lower-order schemes (e.g., Godunov scheme) are generally free of oscillations, but they are so dissipative as to wash out many details. There is therefore a need to develop more advanced schemes that can adequately represent these shocks and discontinuities. Of the many candidate advanced schemes, the total variation diminishing (TVD) scheme is used in the present paper (Tanaka, 1993, 1994).

\section{Simulation Model}

We solve the following 9 set of MHD equations, written in the conservation-law form, as an initial value problem:

$$
\frac{\partial \boldsymbol{u}}{\partial t}+\frac{\partial \boldsymbol{F}}{\partial x}+\frac{\partial \boldsymbol{G}}{\partial y}+\frac{\partial \boldsymbol{H}}{\partial z}=\boldsymbol{S}
$$

Here, $\boldsymbol{F}, \boldsymbol{G}$, and $\boldsymbol{H}$ are respectively the flux functions in the $x, y$, and $z$ directions. The state vector of nine dependent variables is

$$
\boldsymbol{u}=\left(\rho, m_{x}, m_{y}, m_{z}, B_{x}, B_{y}, B_{z}, U, \rho_{2}\right)^{T},
$$

where $\rho$ is the total ion density which is equal to $\rho_{1}+\rho_{2}$ (with $\rho_{1}$ and $\rho_{2}$ respectively corresponding to solar wind $\mathrm{H}^{+}$ions and ionospheric $\mathrm{O}^{+}$ions), $\boldsymbol{m}=\rho \boldsymbol{V}=\left(m_{x}, m_{y}, m_{z}\right)^{T}$ is the momentum, $\boldsymbol{B}$ is the magnetic field, and $U$ is the total energy density. For a two-component plasma, three sets of continuity equations can be written for $\rho, \rho_{1}$ and $\rho_{2}$, and any two of these three continuity equations can be selected as the basic equations. In this paper, therefore, we constructed the basic equations by selecting the continuity equations for $\rho$ and $\rho_{2}$.

The source term $\boldsymbol{S}$ depends on the production rate $q_{1}$ of $\mathrm{H}^{+}$ ions, $q_{2}$ of $\mathrm{O}^{+}$ions (due to photo-ionization and ion-chemical reactions), loss rate $L_{1}$ of $\mathrm{H}^{+}$ions, and $L_{2}$ of $\mathrm{O}^{+}$ions (due to ion-chemical reactions), as well as on the gravitational acceleration $\boldsymbol{g}$ and ion-neutral collision frequency $v$ :

$$
\boldsymbol{S}=\left|\begin{array}{l}
q_{1}+q_{2}-L_{1}-L_{2} \\
-v \boldsymbol{m}-\rho \boldsymbol{g} \\
0 \\
-\frac{\boldsymbol{m}}{\rho} \cdot(v \boldsymbol{m}+\rho \boldsymbol{g})+R_{2} T_{q} \frac{\left(q_{1}+q_{2}\right)}{(\gamma-1)}-R_{2} T_{L} \frac{\left(L_{1}+L_{2}\right)}{(\gamma-1)} \\
q_{2}-L_{2}
\end{array}\right|
$$

In the above formulae $T_{q}=10^{3 \circ} \mathrm{K}$ is the temperature of photo-ions when they are produced, $T_{L}=P /\left(R_{2} \rho\right)$ is the temperature of $\mathrm{O}^{+}$ions when they are lost through the ionchemical reaction with $\mathrm{CO}_{2}, P$ is

$$
P=(\gamma-1)\left(U-\frac{m^{2}}{2 \rho}-\frac{B^{2}}{2}\right)
$$

with $\gamma=5 / 3$ the ratio of specific heats, $R_{2}$ is the gas constant for $\mathrm{O}^{+}$ions, and ion-neutral collision frequency is given as $v=1 \times 10^{-9}\left\{[\mathrm{O}]+\left[\mathrm{CO}_{2}\right]\right\} \mathrm{sec}^{-1}$. The chemical reaction is ignored for $\mathrm{H}^{+}$ions $\left(q_{1}=L_{1}=0\right)$. Loss rate of $\mathrm{O}^{+}$ions is given by $L_{2}=9.4 \times 10^{-10}\left[\mathrm{CO}_{2}\right] \rho_{2}$.

In the present calculation, a neutral atmospheric model is required for three reasons: (1) to calculate photo-ionization rates due to solar EUV, (2) to calculate ion-loss rates due to ion-chemical reactions, and (3) to calculate ion-neutral collision frequencies. It is assumed that the neutral atmosphere consists of oxygen atoms and of carbon dioxide molecules, both of which are stratified gravitationally with a constant temperature $T_{n}=300^{\circ} \mathrm{K}$. It is also assumed that distributions of neutral species are spherically symmetric and do not depend on local time and latitude.

All the calculations are done after normalizing the equation system by planetary radius + altitude of the bottom of the thermosphere $R_{\mathrm{p}}$, solar wind sound velocity $C_{\mathrm{sw}}$, solar wind density $\rho_{\mathrm{sw}}$, and IMF magnitude $B_{\mathrm{sw}}$. Remaining parameters in the calculation are Mach number $M$ and plasma $\beta$ of the solar wind, normalized gravitational acceleration $\boldsymbol{g}$, and neutral densities at the lower boundary. In the present calculation, we assume that $M=6.6$ and $\beta=0.6$, and $\boldsymbol{g}$ is set to the value of Venus. In the case of Mars, $\boldsymbol{g}$ reduces to $1 / 3$ of Venus value. Altitude profiles of neutral densities adopted in the present study are shown in Fig. 1.

Production rate $q_{2}$ is also normalized by $\rho_{\mathrm{sw}}$. After normalizations, the effective rate of solar EUV ionization is same for Mars and Venus, if both of the solar wind density and the solar EUV flux decrease with the square of the distance from the Sun. In the following section, results are given for two cases of effective solar EUV ionization. In case 1, the effective solar EUV ionization is set so that the peak ionospheric plasma pressure is below the incident solar wind dynamic pressure. The effective solar EUV ionization in case 2 is set to be four times as large as that in case 1 . The corresponding solar EUV value for case 2 roughly coincides with that of the solar maximum period.

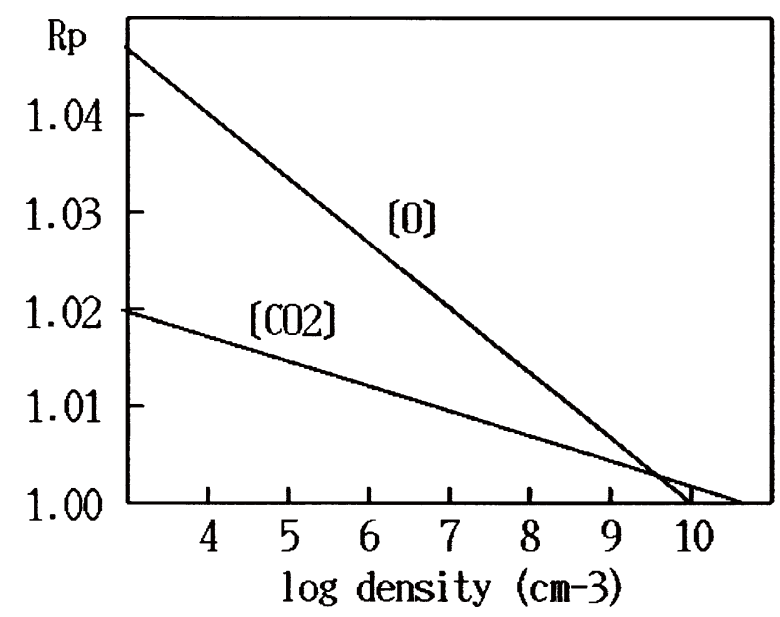

Fig. 1. Altitude profiles of neutral densities adopted in this study. 
In this paper, molecular ions in the lower part of the ionosphere are totally neglected. Thus, ionospheric profiles may not be correct in the bottomside region. Particularly, the results may not be fully applicable to Mars due to the dominance of molecular ions in the ionosphere. However, the dynamical aspect of the interaction process treated in this paper is not severely affected by the distribution of molecular ions.

In the present problem, two different regions having quite different space scales, the solar wind and the ionosphere, must be treated simultaneously. This difficulty can be resolved by using a finite-volume method (FVM). In this method, the integration form of Eq. (1),

$$
\frac{\partial}{\partial t} \int \boldsymbol{u} d v+\int T^{-1} \boldsymbol{F}(T \boldsymbol{u}) d s=\int \boldsymbol{S} d v
$$

is used as the basic equation. Here $d v$ and $d s$ are the volume and surface elements of the control volume, and $T$ is a matrix which rotates the $x$ axis to the direction normal to the control volume surface. The TVD scheme with the monotonic upstream scheme for conservation laws (MUSCL) approach is used for the evaluation of the numerical flux at the interface of the control volume. Details of the present approach are shown elsewhere (Tanaka, 1994; Tanaka and Murawski, 1997).

The equation is solved numerically in all three spatial dimensions $(x, y$, and $z$ ) on a grid system aligned along the spherical $(r, \theta$, and $\phi)$ coordinate. The inner and outer boundaries of the simulation region were set at $1 R_{\mathrm{p}}$ and 10 $R_{\mathrm{p}}$, with $R_{\mathrm{p}}$ the planetary radius + altitude of the bottom of the thermosphere. The simulation code is used on $88 \times 80 \times$ 86 grid (in r, $\theta$, and $\phi$ dimensions). This corresponds to the angular grid spacings $\Delta \theta=2.3^{\circ}$ and $\Delta \phi=4.1^{\circ}$. A nonuniform radial grid was chosen with a finest grid of $0.0025 R_{\mathrm{p}}$ at the inner boundary and a coarsest grid of $0.41 R_{\mathrm{p}}$ at the outer boundary. In the radial direction, therefore, about $1 / 3$ of the total number of grid points were allocated in the ionosphere.

The $x$-axis of our coordinate system is directed antisunward or along the solar wind flow. The solar wind flow impacting the planetary ionosphere is assumed to be parallel to the Sun-Venus line $(-x)$. We also assume that the IMF lies in the equatorial $(x-y)$ plane and is perpendicular to the solar wind flow. Without loss of generality, it is assumed here that the IMF points toward the $+y$ axis (direction of the orbital motion), while the north pole of the planet is in the $+z$ direction. Whereas these solar wind boundary conditions are maintained on the dayside of the outer boundary, zerogradient boundary conditions are maintained on the downstream side.

Near the inner boundary the ion-neutral collision and ionchemical processes become dominant. Therefore, the ionosphere is assumed to be in photochemical equilibrium at the lower boundary. Also, zero plasma velocity is assumed at the inner boundary. The ion temperature at the inner boundary is held constant throughout the simulation process.

A typical computation begins with the boundary conditions written above, selecting desired solar wind values on the outer boundary of the numerical box in the dayside. The numerical solution then continues until the interaction pro- cess achieves an approximately steady state. Initial conditions for the solar wind velocity outside $1.1 R_{\mathrm{p}}$ were derived from the potential flow past a sphere. Similarly, the IMF in this region was derived from the potential magnetic field around a sphere. The initial conditions for density and temperature in the solar wind region were constant everywhere. In the ionospheric region below $1.1 R_{\mathrm{p}}$, initial conditions for $\boldsymbol{m}$ and $\boldsymbol{B}$ were equal to zero. Assuming a proper peak height, the initial ion density in the ionosphere was derived from the photo-chemical equilibrium condition below the peak height and from the gravitational stratification above the peak height. Initially the ionospheric temperature was equal to $1000^{\circ} \mathrm{K}$ everywhere. The normalization time unit $t_{\mathrm{n}}$ is given by $R_{\mathrm{p}} / C_{\mathrm{sw}}$. With a typical solar wind condition, $1 t_{\mathrm{n}}$ roughly corresponds to $100 \mathrm{sec}$. The structure of the solar wind flow reaches a stationary state after about $10 t_{\mathrm{n}}$. However, it requires much more time for the ionosphere to reach a stationary state, because plasma flow is very slow in the ionosphere. In this paper, numerical results are shown after an integration of $100 t_{\mathrm{n}}$.

\section{Results and Discussion}

Plate 1 shows by color codes the two-dimensional (2-D) distributions of four plasma variables, $\log \left(\rho / \rho_{\mathrm{sw}}\right), \log \left(\rho_{2} / \rho_{\mathrm{sw}}\right)$, $P / P_{\mathrm{sw}}$, and $B / B_{\mathrm{sw}}$, in the sun-planet meridian plane (top half) and in the equatorial plane (bottom half), for case 1. Plate 2 shows similar results for case 2. In Plates 1 and 2, the solar wind impinges on the planet from the left. Global features of the solar wind-planetary ionosphere interaction can be observed from these plates. The formation of the bow shock is clearly seen as discontinuities in total density, pressure and magnetic field. By comparing the total and $\mathrm{O}^{+}$densities, we can see that plasma in the bow shock region consists of $\mathrm{H}^{+}$ ions. Concerning discussions about the altitude of the bow shock and its asymmetry between the equatorial and meridian planes, see Tanaka and Murawski (1997).

Both in cases 1 and 2, the magnetic field increases monotonically to a peak in the magnetosheath downstream of the bow shock, but in case 2 eventually vanishes on the downstream side near the planetary surface, creating a diamagnetic region. While this monotonic increase of the magnetic field corresponds to the formation of the magnetic barrier (Zhang et al., 1991), the appearance of a diamagnetic cavity in case 2 is due to the formation of the ionopause, which forms where the impacting solar wind pressure is balanced by the ionospheric pressure (Luhmann et al., 1987). In case 1 , on the other hand, the formation of the ionopause is not so clear as it is in case 2. No diamagnetic cavity is observed in this case. In case 2 , the altitude at which the ionopause is located is smallest at the nose and increases monotonically with increasing solar zenith angle (SZA), reaching a maximum altitude at the terminator.

High-density and high-pressure regions around the planet correspond to the ionosphere and the ionotail. Close to the planetary surface and in the tail region, the profiles of the total mass density are very similar to the corresponding profiles of $\mathrm{O}^{+}$ions. We therefore deduce that the ionospheric and ionotail plasma consists primarily of cold and dense $\mathrm{O}^{+}$ ions. On the nightside the ionotail exhibits rather complex structures which consist primarily of $\mathrm{O}^{+}$ions. The complex 

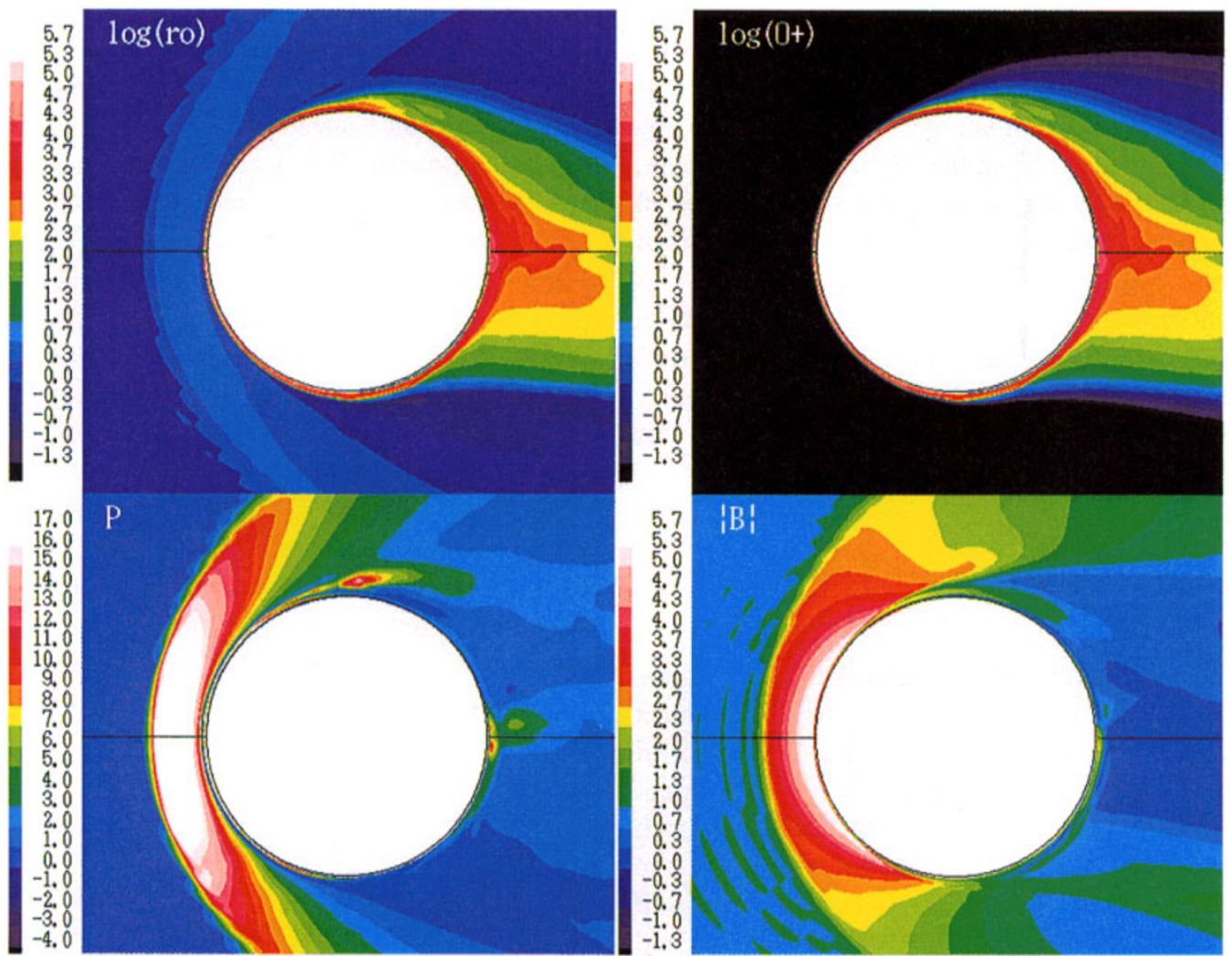

Plate 1. Distributions of plasma variables in the meridian and equatorial planes, for case 1. The top half of each panel corresponds to values in the meridian plane while the bottom half corresponds to values in the equatorial plane. The top left panel is a plot of log total mass density, the top right is $\log \mathrm{O}^{+}$ion density, the lower left is pressure, and the lower right is magnetic field magnitude. All the variables have been normalized with solar wind parameters. Note the bow shock consists of $\mathrm{H}^{+}$ions and the ionosphere consists of low-temperature and high-density $\mathrm{O}^{+}$ions.
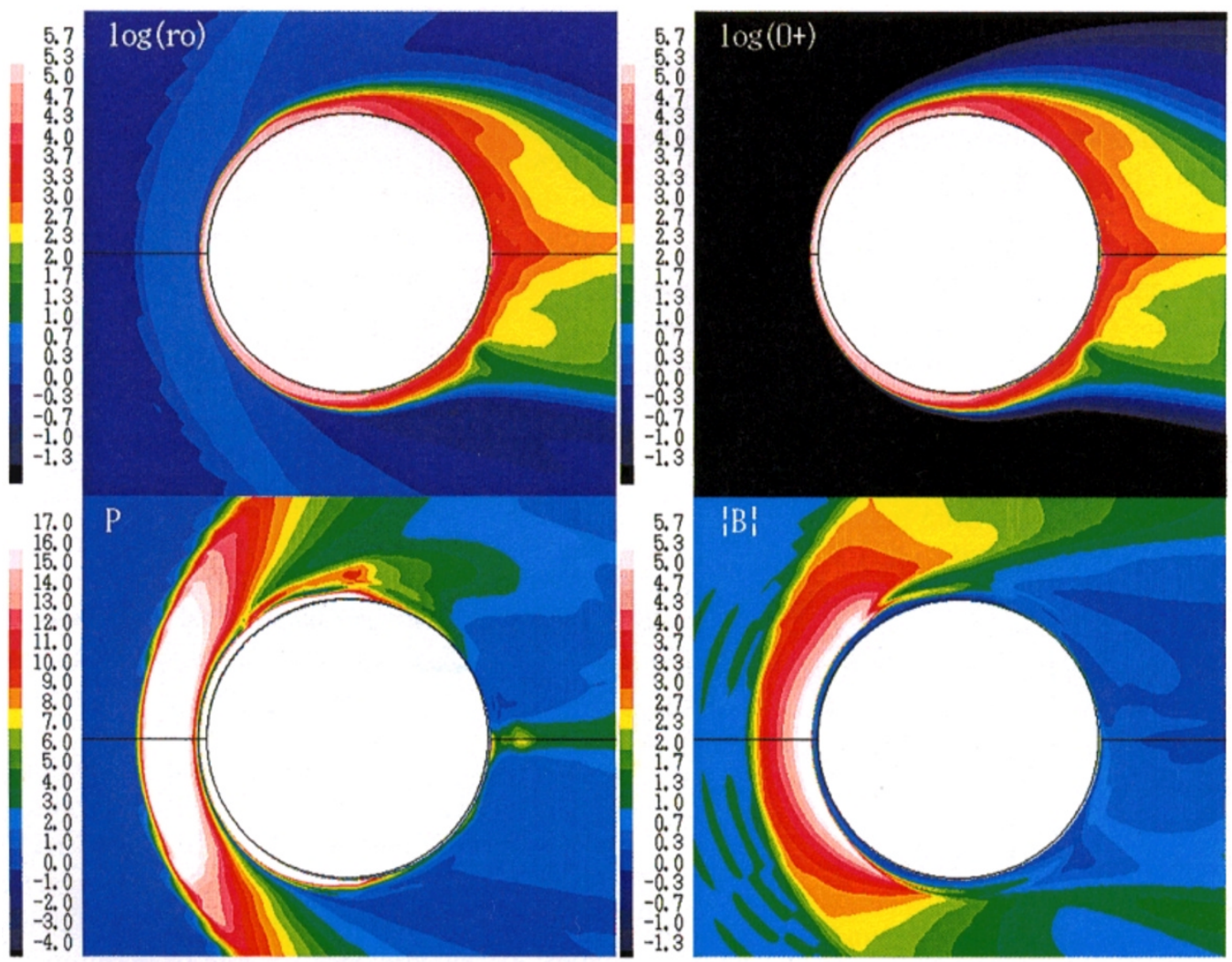

Plate 2. Same as Plate 1, but for case 2. 


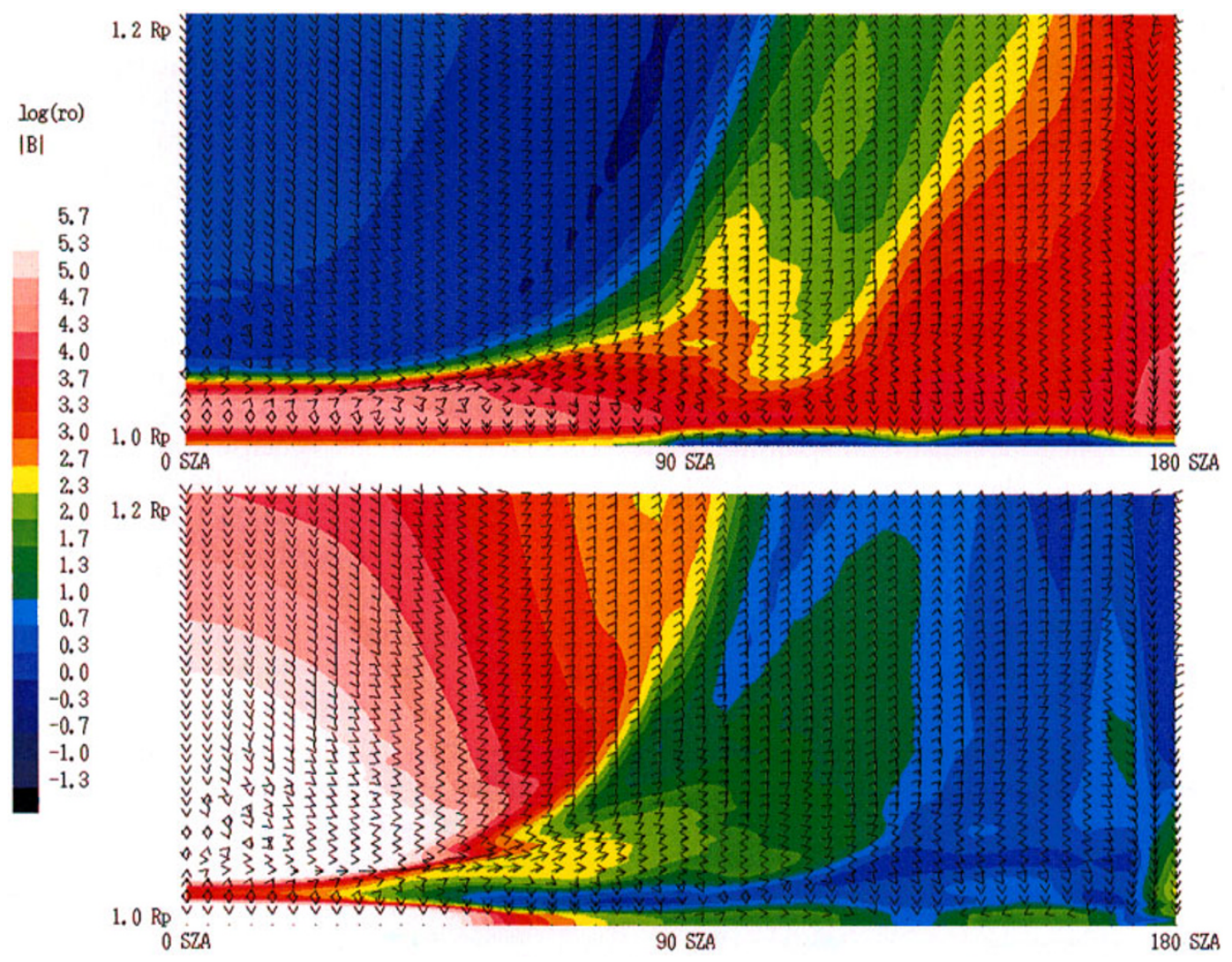

Plate 3. Distributions of normalized density $\log \left(\rho / \rho_{\mathrm{sw}}\right)$ (top panel) and normalized magnetic field magnitude $B / B_{\mathrm{sw}}$ (bottom panel) with normalized mass flux $\rho V / \rho_{\mathrm{sw}} V_{\mathrm{sw}}$ (arrows), in the meridian plane for case 1. Two dimensional distributions are shown by color levels as functions of the solar zenith angle (SZA, horizontal axis) and the altitude (vertical axis). The vertical axis is enlarged about ten times compared with the horizontal axis.

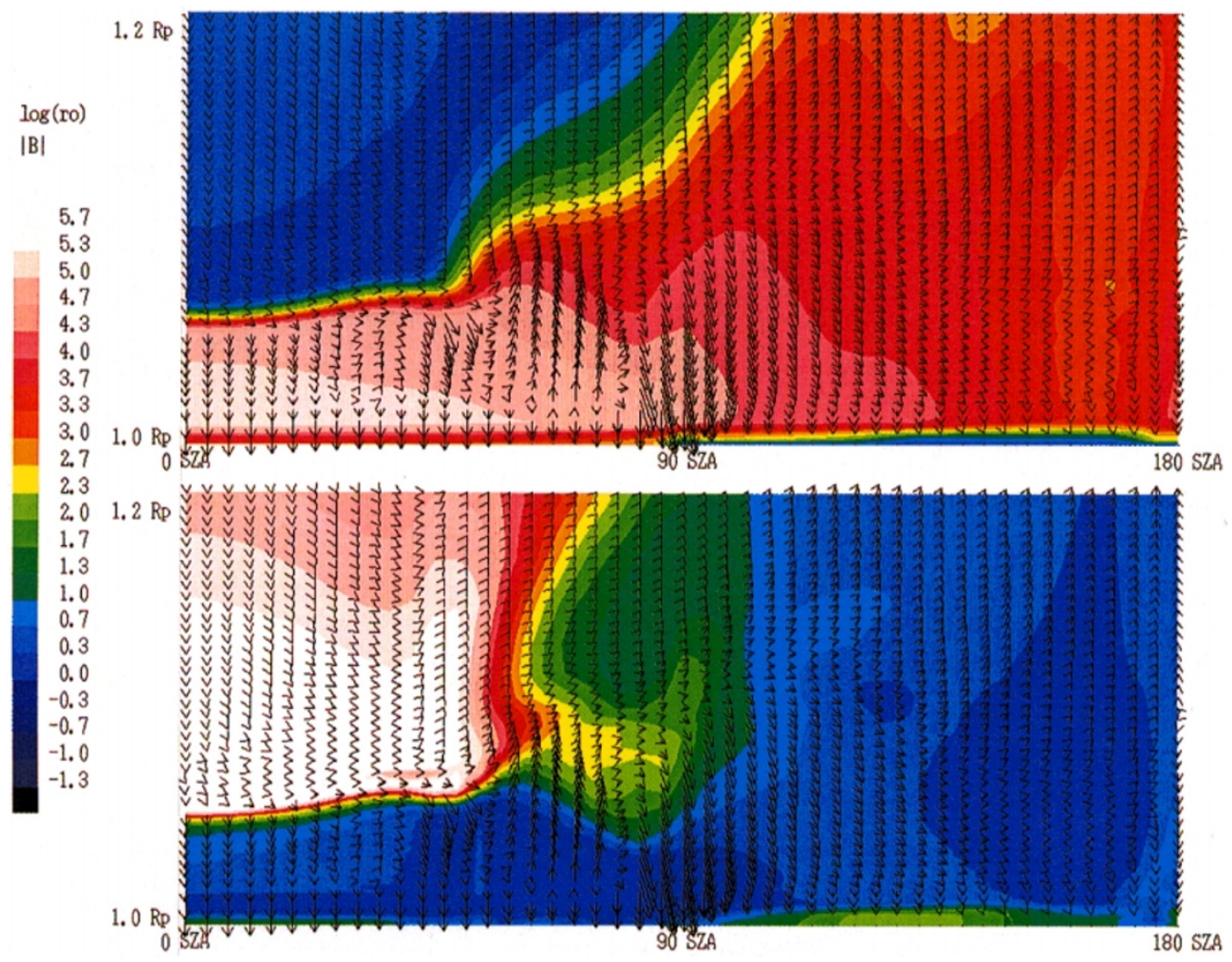

Plate 4. Same as Plate 3, but for case 2. 
structures in the nightside region were already discussed by Tanaka and Murawski (1997), who pointed out the role of the draped IMF for the formations of the wing structures in the ionotail and the ionospheric holes. The dayside ionosphere is thicker in case 2 than in case 1 reflecting the difference in the amount of the solar EUV flux. However this difference in $\mathrm{O}^{+}$ion density is not so clear in the ionotail region. Comparing results for cases 1 and 2, plasma densities in the ionotail are quite similar with each other. This point will be discussed later concerning the results shown in Plates 3 and 4.

The pressure and magnetic field distributions in the topside ionosphere of Plates 1 and 2 show rather complex structures in the meridian planes from 45 to 90 SZA. In this area, the draping IMF slipping over the poles strongly interacts with the underlying ionosphere. Partial penetration of the magnetic field into the topside ionosphere and simultaneous leaping up of the pressure are clearly seen in the meridian planes of Plates 1 and 2 . The regions of penetrating magnetic field extend into the nightside as yellow/green strips. These processes suggest an important role of non-ideal MHD effects around there, because the mixture of solar wind magnetic field and ionospheric plasma is occurring. In the equatorial plane, on the other hand, the interaction surface between the IMF and the underlying ionosphere is distributing smoothly from the dayside to the nightside.

The basic structure of the solar wind-ionosphere interaction in the subsolar region can be well understood from Fig. 2, which shows the distributions of kinetic (RAM), gas (P), and magnetic pressures (B) normalized by $P_{\mathrm{sw}}$ along the Sunplanet line. In Fig. 2, the upper panel shows the result for case 1 and the lower panel shows the result for case 2 . The small squares on the curves in Fig. 2 indicate the positions of radial grid points. A dense grid is seen in the ionosphere, while a coarse grid is seen at the upstream region. The gas pressure dominates over the magnetic and kinetic pressures in the upper magnetosheath region (right side). Closer to the planetary surface (left side) the magnetic pressure piles up against competitive ionospheric gas pressure, while at the same time gas pressure decreases. This behavior is a consequence of the formation of the magnetic barrier located at the maximum of the magnetic pressure. The strongest magnetic field at the magnetic barrier reaches a value of approximately $7.5 \times B_{\mathrm{sw}}=103 \mathrm{nT}$.

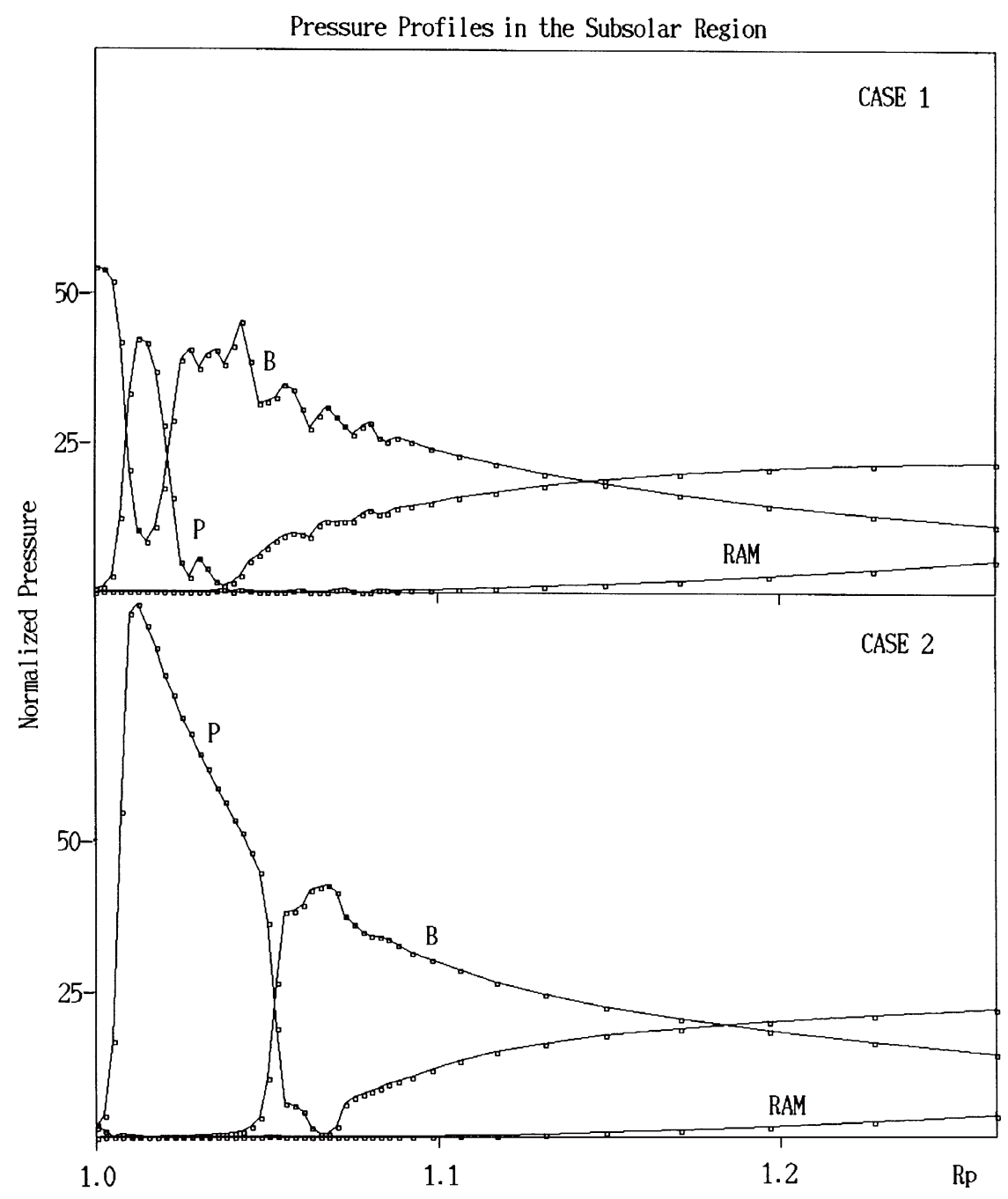

Fig. 2. The distributions of the gas pressure (P), the magnetic pressure $B^{2} / 2(\mathrm{~B})$, the dynamic pressure $\rho V^{2}$ (RAM) along the Sun-planet line, for case 1 (upper) and for case 2 (lower). These three variables are normalized by solar wind pressure. The horizontal axis shows the radial distance normalized to $R_{\mathrm{p}}$ (planetary radius) and the vertical axis shows normalized pressure values. 
In case 2 , the gas pressure of cold ionospheric plasma supports the major part of the magnetic barrier forming the ionopause. This result corresponds to the case of an unmagnetized ionosphere (Shinagawa, 1996a). The ionospheric pressure is maintained by photo-ionization and ionchemical processes in the planetary upper atmosphere. At the bottom of the ionosphere, ionospheric gas pressure is supported against gravity by the neutral atmosphere through ion-neutral collisions. It is noteworthy that the structure of the neutral atmosphere is very important for ionospheric dynamics, primarily as the factor controlling vertical diffusion, and secondarily as the cause of the drag force exerted on ions moving horizontally. All of these effects are basically included in the present simulation model.

Even in case 2, numerical dissipation, which is unavoidable in any numerical simulation, may cause small penetration of the magnetic field into the diamagnetic cavity. However, the amount of the penetration into the ionosphere is very small in the lower panel of Fig. 2, indicating that the dissipation effect which comes from numerical viscosity included in the TVD scheme is very small in the sub-solar region. In the upper panel of Fig. 2 (case 1), on the other hand, penetration of the magnetic field into the ionosphere is apparent. In this case, the highly magnetized ionosphere as shown by Shinagawa (1996b) is realized. Pressure and magnetic field gradients in the interface region are sharper in case 2 than in case 1 . Lower order numerical schemes may not be able to adequately capture this discontinuity. Contrary to the calculation of Shinagawa (1996b), the diffusion effect is not effective in the present calculation. Vertical convection induced from pressure imbalance in a non-stationary state can cause redistribution of magnetic flux from the magnetic barrier to the ionosphere, without diffusion effect. Through this process, the solar wind IMF penetrates the ionosphere and supplements the total pressure with magnetic pressure in a stationary state. These redistributions extend even to the bottomside of the ionosphere. The bottomside magnetic field thus generated will be observed as remnant field even if relative ionospheric pressure increases again to a level sufficient to support the solar wind (Russell et al., 1983). In Fig. 2, peak ionospheric density in case 1 is only $1 / 2$ of that in case 2, despite the fact that solar EUV flux in case 1 is reduced to $1 / 4$ of that in case 2 . These results indicate that the penetrated IMF not only supplements the pressure but also

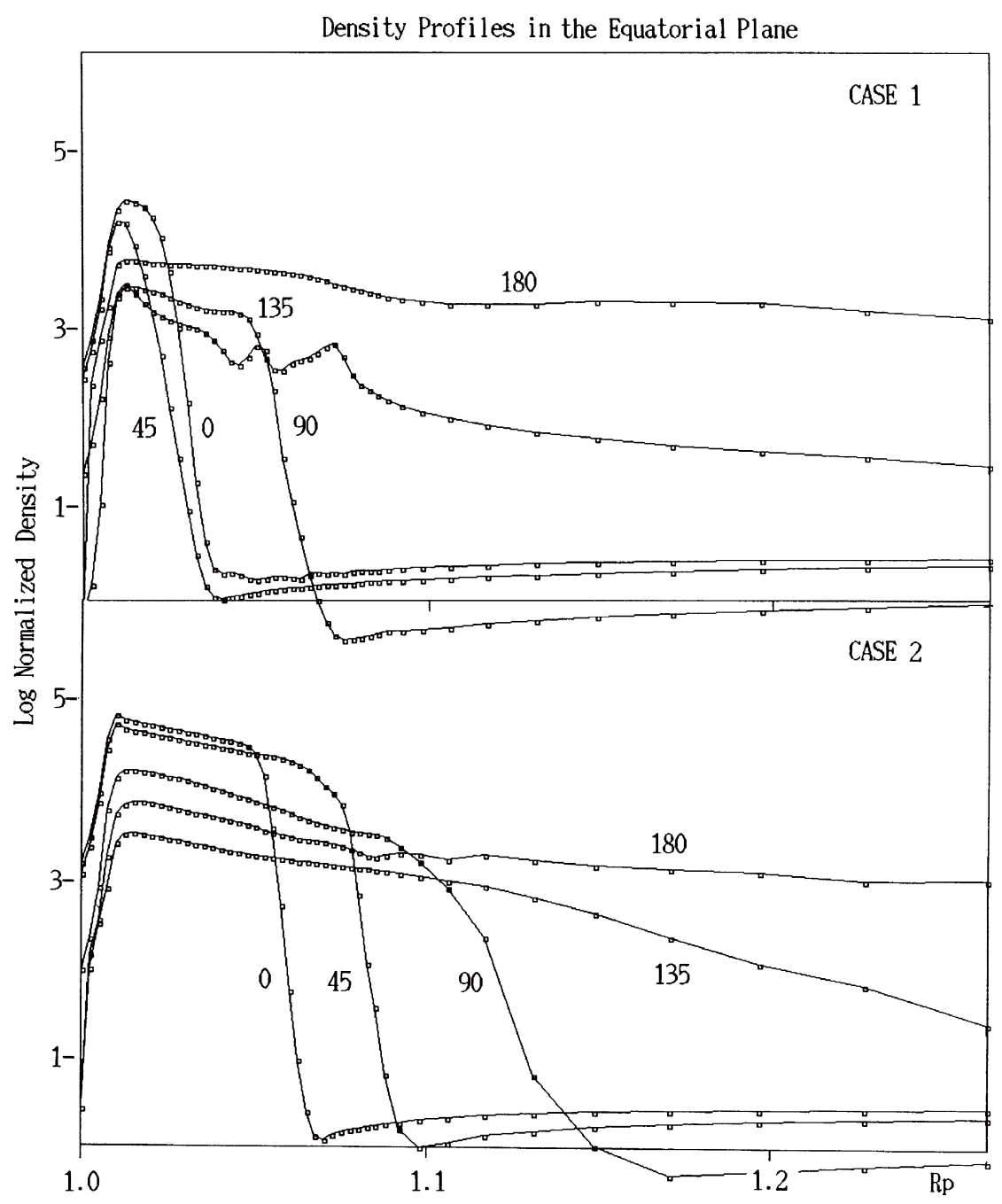

Fig. 3. Log plots of the altitude distributions for the normalized density along radial lines of constant solar zenith angle (SZA), for case 1 (upper) and for case 2 (lower). Note the anomalous profiles near the terminator (for 90 and 135 SZAs) for case 1, and the relatively sharp ionopause on the subsolar side (for 0 and 45 SZAs) for case 2. In the tail region (for 135 and 180 SZAs) there are extended regions of mass density enhancements corresponding to the tail rays. 
affects the vertical transport of the ionospheric plasma.

Figure 3 shows some altitude profiles of $\mathrm{O}^{+}$ion density normalized by $\rho_{\mathrm{sw}}$ at different SZAs in the equatorial plane. In this figure, the upper and lower panels show results for case 1 and case 2 respectively, with the vertical axis being plotted in a log scale. In case 2, the dayside ionosphere for 0 and 45 SZAs exhibits a gravitational stratification in the main part and a sharp decrease in the topside region. This sharp cutoff in the topside density distribution is a manifestation of the ionopause. In case 1, on the other hand, the density distributions of the dayside ionosphere no longer show normal stratifications. At the terminator for 90 SZA and in the nightside for 135 and 180 SZAs, the ionopause is less pronounced both in cases 1 and 2 . In these regions, the ionosphere extends to high altitudes, although density distributions in the main part of case 2 still exhibit gravitational stratifications.

Comparing with case 2, results for case 1 shown in the upper panel of Fig. 2 exhibit anomalous features. The scale height of the terminator ionosphere for 90 SZA shows a departure from a gravitational stratification with irregular variations of the density distribution in the topside. This irregular variation in the density distribution is also observed in the result for 135 SZA. While the peak ionospheric density in case 2 shows a monotonous decrease toward night until 135 SZA, the peak ionospheric density in case 1 for 135 SZA becomes greater than that for 90 SZA. These anomalous features in the ionospheric density distribution are due to horizontal magnetic field which prevents the vertical diffusion of plasma. It is seen from Plate 1 that the terminator ionosphere in case 1 is imbedded in the region of penetrated IMF. In both cases 1 and 2, the midnight ionospheric density becomes higher than that of 135 SZA. These results correspond to the formation of the ionotail.

The 2-D distributions of $\log \left(\rho / \rho_{\mathrm{sw}}\right)$ (top) and $B / B_{\mathrm{sw}}$ (bottom) by color codes and flux $\rho \boldsymbol{V} / \rho_{\mathrm{sw}} V_{\mathrm{sw}}$ by arrows in the meridian plane are shown in Plate 3 for case 1 , where the $x$ and $y$ axes are the SZA and the altitude. The vertical ( $y$ ) axis of this figure is enlarged about ten times compared with the horizontal $(x)$ axis. Therefore vertical components of the arrows are also enlarged ten times in order to show relatively correct directions along color distributions. Plate 4 is similar to Plate 3, but for case 2. In Plates 3 and 4, regions with blue color in the density distribution and with white/red color in the magnetic field distribution correspond to the solar wind. It can be generally noted in Plates 3 and 4 that density and magnetic field tend to exclude with each other. This mutual exclusion is most clear in the dayside part of Plate 4. Around there, the ionopause is seen as sharp gradients of $\log \left(\rho / \rho_{\mathrm{sw}}\right)$ and $B / B_{\text {sw }}$. In Plate 3 , on the other hand, mixing of ionospheric plasma and solar wind magnetic field is apparent in the dayside ionosphere.

The dayside ionosphere for case 2 shown in Plate 4 is a diamagnetic ionosphere. In the lower panel of Plate 4 , the strong $\mathrm{B}$ region (white color) just above the dayside ionopause is the magnetic barrier. Out of the magnetic barrier, a beltlike structure with a yellow/green color for B is extending from 60 SZA to 90 SZA. This structure corresponds to the partial penetration of magnetic field which causes the mixture of the solar wind IMF and ionospheric plasma (also see Plate
2). As a consequence, the ionopause structure becomes obscure in the region with SZA greater than 45. In the course of partial penetration, the draped IMF pushes the terminator ionosphere downward. In case 2, however, the resisting action of the terminator ionosphere against IMF penetration is still sufficient to make the main part of the nightside ionosphere remain weakly magnetized. As for the ionospheric plasma motion, the arrow distributions in Plate 4 show that the down-welling ionospheric flow is generated corresponding to a lower ionopause height. This flow structure is due to the influence of pressure imbalance caused by magnetic tension force exerting on the ionopause.

In a previous 2-D model given by Shinagawa (1996b), effects of the solar wind on the plasma transport process in the ionosphere were investigated considering only magnetic pressure of the overlying magnetic barrier. The present 3-D model treats the interaction process between the solar wind and the ionosphere more generally, taking into consideration the effects of the solar wind dynamic pressure and magnetic tension. It is apparent in the present results that the impinging solar wind exerts severe dynamical controls on ionospheric plasma transport. In Plate 4, horizontal flow in the dayside and terminator ionosphere is supplying plasma from the dayside to the nightside. However, this flow is affected by the penetrating magnetic field and by pressure imbalance generated through the interaction between the solar wind and the ionosphere. Down-welling motion around 90 SZA is correlated with the distribution of penetrating IMF, although a complete stationary state is not yet realized around there. It may require much longer time to get a complete stationary state for the result shown in Plate 4.

The midnight ionosphere and ionotail regions seem to be magnetized very weakly. However, the mechanism of weak magnetization in the ionotail is different from that in the dayside ionosphere. The ionotail regions coincide with the neutral sheet of the draping IMF. Penetration of the ionospheric plasma into the neutral sheet generates weakly magnetized regions in the nightside (Tanaka and Murawski, 1997). The weakly magnetized region with a red color in the density and a blue color in magnetic field distribution around midnight is a consequence of ionotail formation.

Contrary to case 2, the solar wind magnetic field penetrates to the main part of the dayside ionosphere in case 1. However, the lower panel of Plate 3 indicates that partial penetration of solar wind magnetic field from the magnetic barrier also occurs even in case 2 . A belt-like region whose color for B changes from yellow to green extends deep into the nightside. In the region of 90-135 SZA, this belt-like region in the $B$ distribution is overlapping with a yellow/ green region in the density distribution and acts to choke the terminator ionosphere. As a result, low-density ionosphere is generated around the region of 90-135 SZA. The flow of $\mathrm{O}^{+}$ion in this region is controlled by magnetic forces and directed horizontally. In case 1 , the ionotail region is a continuation from a narrow horizontal belt of the weakly magnetized ionosphere in the terminator. Despite the lowdensity ionosphere for 90-135 SZA in case 1, plasma density in the ionotail for case 1 is quite similar to that of case 2 (also see Plates 1 and 2). The plasma density in the ionotail is out of proportion to that in the dayside and 


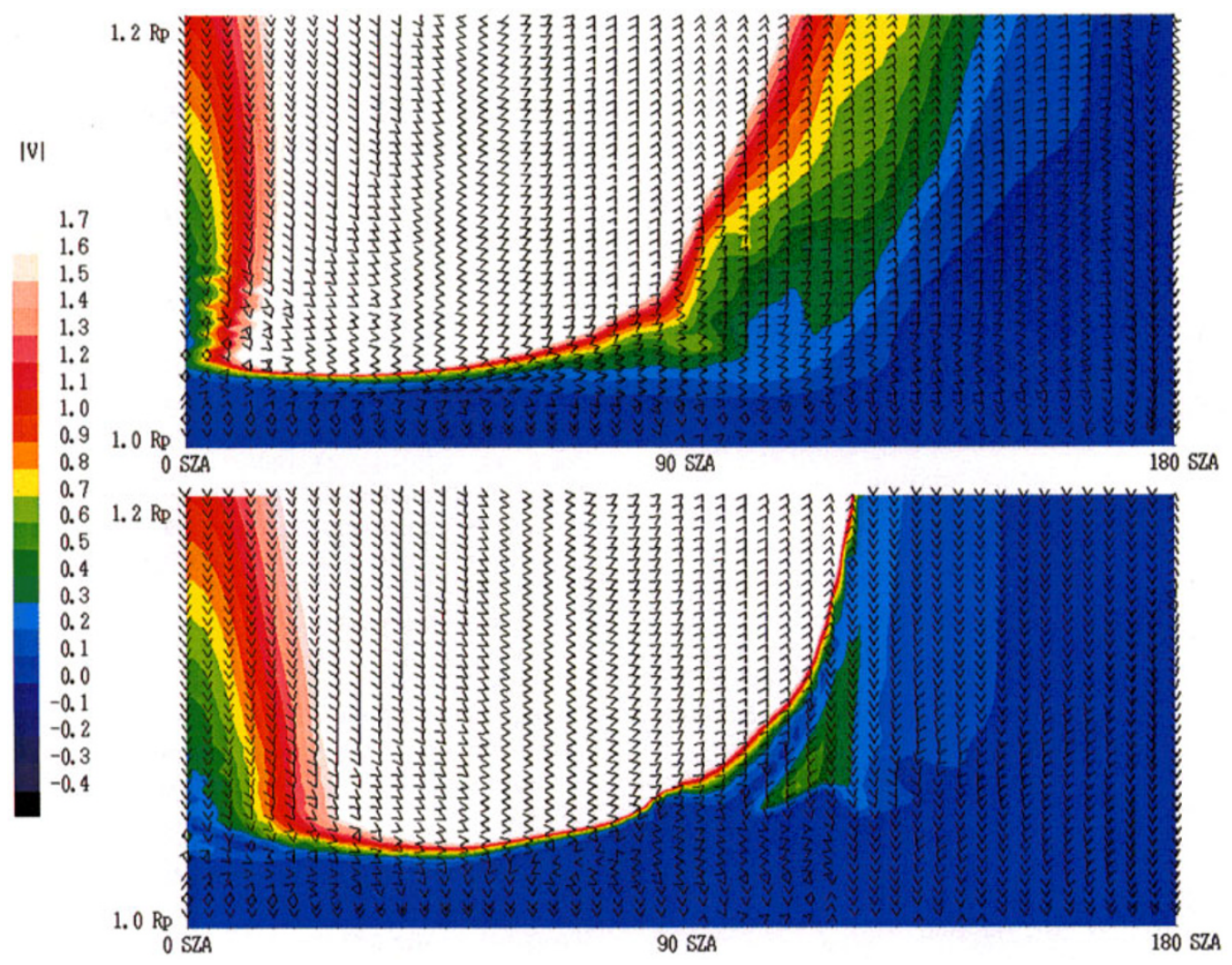

Plate 5. Distributions of normalized velocity $V / V_{\mathrm{sw}}$ in the meridian (top panel) and equatorial (bottom panel) planes for case 1, with normalized mass flux $\rho \boldsymbol{V} / \rho_{\mathrm{sw}} V_{\mathrm{sw}}$ shown by arrows. Two dimensional distributions are shown by color levels as functions of the solar zenith angle (SZA, horizontal axis) and the altitude (vertical axis). The vertical axis is enlarged about ten times compared with the horizontal axis.

terminator ionospheres. This result is related to the fact that the formation of the ionotail is strongly controlled by the draping magnetic field. A reservoir effect of the ionotail can be expected from this result.

Plate 5 shows by color codes 2-D distributions of $V / V_{\text {sw }}$ in the meridional (top) and equatorial (bottom) planes, for case 1. The format used for Plate 5 is the same to that for Plates 3 and 4. In Plate 5, high (white color) and low (blue color) velocity regions correspond to the solar wind and the ionosphere, respectively. An exception to this correspondence is a slow velocity region seen in the solar wind around 0 SZA. The flow structure in this region was already shown in Fig. 2.

Flow velocity in the dayside ionosphere is very small both in the meridian and equatorial planes, despite the penetration of the IMF to the dayside ionosphere. This result indicates that the IMF supplements only static pressure in the dayside. In the terminator and nightside ionospheres, on the other hand, features seen in the meridian (upper) and equatorial (lower) planes are different from each other. In the equatorial plane, high (white color) and low (blue color) velocity regions separately occupy the solar wind and the ionosphere respectively, with a clear border between them. In this plane, a green spot in the ionosphere is not due to the solar wind flow but due to the return flow in the ionotail. In the meridian plane, green/magenta regions prevail between the solar wind and the ionosphere. This green/magenta region corresponds to the place where partial penetration of the IMF is occurring. In this region, the flow velocity gradually decreases from the solar wind to the ionosphere. However, it seems interesting to note that the flow velocity is nearly constant along the path of IMF penetration (see lower panel of Plate 3).

\section{Conclusion}

The effects of decreased ionospheric pressure on the solar wind-interaction with non-magnetized planets have been investigated from numerical MHD simulations. When the peak ionospheric plasma pressure decreases below the incident solar wind dynamic pressure, the IMF penetrates from the magnetosheath to the dayside ionosphere so as to adjust self consistently the ionospheric total pressure. This result for the pressure balance in the dayside ionosphere is quite consistent with the experimental results shown by Luhmann et al. (1987). The process of IMF penetration does not require a magnetic diffusion effect. It is a general belief that low ionospheric pressure condition usually prevails at Mars, although at Venus only when the incident solar wind pressure is extremely high. Penetrated IMF also affects the vertical distributions of the terminator and nightside ionospheres. In these areas, anomalous ionospheric stratifications are caused due to the suppression of vertical plasma motion.

Contrary to the dayside, decreased ionospheric pressure does not directly result in the decrease of ionotail plasma density. The formation process of the ionotail is mainly controlled by the draping IMF. In this configuration, a reservoir effect of the ionotail tends to weaken a change of 
plasma density.

An important process predicted from the present study is partial penetration of the draping IMF from the magnetic barrier to the terminator ionosphere. This process characterizes the interaction between the draping IMF and the ionopause, and must be a consequence of nonideal MHD effects. Heikkila (1997) investigated a nonideal MHD effect acting on the earth's magnetopause, in order to explain the impulsive transfer of solar wind plasma through the magnetopause. The present problem is somewhat similar to this concept, although the mutual roles played by plasma and magnetic field are opposite here. These predictions must be confirmed from future planetary missions.

\section{References}

Brace, L. H., W. T. Kasprzak, H. A. Taylor, R. F. Theis, C. T. Russell, A. Barnes, J. D. Mihalov, and D. M. Hunten, The ionotail of Venus: Its configuration and evidence for ion escape, J. Geophys. Res., 92, 15-26, 1987.

Heikkila, W. K., Interpretation of recent AMPTE data at the magnetopause, J. Geophys. Res., 102, 2115-2124, 1997.

Luhmann, J. G., The solar wind interaction with Venus, Space Sci. Rev., 44, 241-306, 1986.

Luhmann, J. G. and L. H. Brace, Near-Mars space, Rev. Geophys., 29, 121140, 1991.

Luhmann, J. G. and T. E. Cravens, Magnetic fields in the ionosphere of Venus, Space Sci. Rev., 55, 201-274, 1991.

Luhmann, J. G., C. T. Russell, F. L. Scarf, L. H. Brace, and W. C. Knudsen, Characteristics of the Marslike limit of the Venus-solar wind interaction, J. Geophys. Res., 92, 8545-8557, 1987.

Phillips, J. L. and D. J. McComas, The magnetosheath and magnetotail of
Venus, Space Sci. Rev., 55, 1-80, 1991.

Phillips, J. L., J. G. Luhmann, and C. T. Russell, Magnetic configuration of the Venus magnetosheath, J. Geophys. Res., 91, 7931-7938, 1986.

Russell, C. T., J. G. Luhmann, and R. C. Elphic, The properties of the low altitude magnetic belt in the Venus ionosphere, Adv. Space Res., 2(10), 13-16, 1983.

Shinagawa, H., A two-dimensional model of the Venus ionosphere 1. Unmagnetized ionosphere, J. Geophys. Res., 101, 26,911-26,919, 1996a.

Shinagawa, H., A two-dimensional model of the Venus ionosphere 2. Magnetized ionosphere, J. Geophys. Res., 101, 26,921-26,930, 1996b.

Slavin, J. A., K. Schwingenschuh, W. Riedler, and Y. Yeroshenko, The solar wind interaction with Mars: Mariner 4, Mars 2, Mars 3, Mars 5 , and Phobos 2 observations of bow shock position and shape, J. Geophys. Res., 96, 11,235-11,241, 1991.

Tanaka, T., Configurations of the solar wind flow and magnetic field around the planets with no magnetic field: calculation by a new MHD simulation scheme, J. Geophys. Res., 98, 17,251-17,262, 1993.

Tanaka, T., Finite volume TVD scheme on an unstructured grid system for three-dimensional MHD simulation of inhomogeneous systems including strong background potential fields, J. Comput. Phys., 111, 381-389, 1994.

Tanaka, T. and K. Murawski, Three-dimensional MHD simulation of the solar wind interaction with the ionosphere of Venus: Results of twocomponent reacting plasma simulation, J. Geophys. Res., 102, 19,80519,821, 1997.

Zhang, T. L., J. G. Luhmann, and C. T. Russell, The solar cycle dependence of the location and shape of the Venus bow shock, J. Geophys. Res., 95, 14,961-14,967, 1990.

Zhang, T. L., J. G. Luhmann, and C. T. Russell, The magnetic barrier at Venus, J. Geophys. Res., 96, 11,145-11,153, 1991.

T. Tanaka (e-mail: tanaka@crl.go.jp) 